\title{
A METRIC CHARACTERIZING ČECH DIMENSION ZERO
}

\author{
K. A. BROUGHAN
}

\begin{abstract}
In this paper we prove the following: a metrizable space $(X, \tau)$ has (Čech) dimension zero if and only if there is a metric for $X$, generating the topology $\tau$, taking values in some subset of the nonnegative real numbers with 0 as its only cluster point.
\end{abstract}

It is possible to categorize some classes of metrizable spaces using the existence of metrics with special properties. See for example J. de Groot [1], J. Nagata [2], and L. Janos [4]. One may also obtain classifications depending on the existence of metrics with values in subsets of the real numbers. For instance, a metrizable space has a metric with values in the nonnegative integers if and only if it is regular $A$ space, [5]. In this note we extend the classification to those spaces having metrics with values in a subset of the positive real numbers with 0 as the only cluster point.

Let $R$ be the set of real numbers and $N$ the positive integers.

Definition. We say $(X, \tau)$ is $F$-metrizable if there exists a metric $\rho$ for $X$, generating the topology $\tau$, such that for all $(x, y) \in X \times X, \rho(x, y) \in$ $F \subset[0, \infty)$. We call such a $\rho$ an $F$-metric. For example, every metrizable space is $F$-metrizable with $F=[0,1]$.

Before coming to the main theorem we prove a special case of it:

TheOREM 1. Let $(X, \tau)$ be metrizable and let $F=\{0\} \cup\left\{1 / 3^{n} \mid n \in N\right\}$. The following are equivalent:

(i) $X$ is F-metrizable,

(ii) $X$ has $\breve{C}$ ech dimension zero, i.e. Ind $X=0$.

Proof. "(ii) $\Rightarrow$ (i)". Let $B(m)$ be the Baire space of weight $m$, that is, the cartesian product of a countable family of discrete spaces each of cardinality $m$.

We will show first that $B(m)$ is $F$-metrizable.

Received by the editors September 19, 1972.

AMS (MOS) subject classifications (1970). Primary 54E35, 54F45.

Key words and phrases. Metric spaces, Cech dimension zero.

(c. American Mathematical Society 1973 
If $x$ and $y$ are distinct points in $B(m)$ let $f(x, y)=\min \left\{k \mid x_{k} \neq y_{k}\right\}$ and define

$$
\begin{aligned}
\rho(x, y) & =3^{-f(x, y)} & & \text { when } x \neq y, \\
& =0 & & \text { otherwise. }
\end{aligned}
$$

Then $\rho$ is an $F$-metric for $B(m)$ generating the topology. Thus $B(m)$ is $F$ metrizable. Now, if $X$ has Cech dimension zero, we can imbed it in $B(m)$ for some $m$ [6]. The result now follows upon making the observation that subspaces of $F$-metrizable spaces are $F$-metrizable.

"(i) $\Rightarrow$ (ii)". Let $\rho$ be an $F$-metric for $X$ generating the topology $\tau$. We will show that $\rho$ is a nonarchimedian metric. Let $x, y$ and $z$ be three distinct points in $X$ and suppose that $\rho(x, y)=1 / 3^{l}, \rho(x, z)=1 / 3^{m}$ and $\rho(x, y)=1 / 3^{n}$. Then $1 / 3^{l} \leqq 1 / 3^{m}+1 / 3^{n}$. If $m \leqq l$ then $1 / 3^{l} \leqq 1 / 3^{m} \leqq$ $\max \left\{1 / 3^{m}, 1 / 3^{n}\right\}$. On the other hand, if $m>l$ and $n>l$, suppose for instance that $m \geqq n>l$. Then

$$
1 / 3^{n}+1 / 3^{n} \geqq 1 / 3^{m}+1 / 3^{n} \geqq 1 / 3^{l} .
$$

Therefore $2 / 3^{n} \geqq 1 / 3^{l}$ and thus $2 \geqq 3^{n-1} \geqq 3$, a contradiction. Thus either $m \leqq l$ or $n \leqq l$. In either case $1 / 3^{l} \leqq \max \left\{1 / 3^{m}, 1 / 3^{n}\right\}$. Therefore $\rho(x, y) \leqq$ $\max \{\rho(x, z), \rho(z, y)\}$. This inequality holds also when the three points are not distinct. Thus $(X, \tau)$ has a nonarchimedian metric and hence (by [1]), Ind $X=0$.

The main result depends upon a special case of the following theorem of J. Nagata [3], "A space $X$ has (Čech) dimension $\leqq n$ if and only if we can introduce in $X$ a topology preserving metric $\rho$ such that all the open balls $B(x, \varepsilon)$ have boundaries of dimension $\leqq n-1$ and such that $\{B(x, \varepsilon) \mid x \in X\}$ is closure preserving for every $\varepsilon>0$."

Let $B=\left\{a_{n}\right\} \cup\{0\}$ where $\left\{a_{n}\right\}$ is an arbitrary strictly monotonically decreasing sequence of real numbers with limit zero and $H=\{1 / n \mid n \in N\} \cup$ $\{0\}$.

Theorem 2. Let $(X, \tau)$ be metrizable. Then the following are equivalent:

(i) Ind $X=0$,

(ii) $X$ is $B$-metrizable,

(iii) $X$ is $\mathrm{H}$-metrizable.

Proof. "(ii) $\Rightarrow$ (iii)" is immediate.

"(iii) $\Rightarrow$ (i)". We will use Nagata's Theorem in the case $n=0$. Let $\rho$ be a compatible metric with values in $\{0\} \cup\{1 / n \mid n \in N\}=H$. Let $p \in X$ and $\varepsilon>0$ be given. We will show firstly that $B(p, \varepsilon)$ has an empty boundary. This follows from the following:

LemMa. $S(p, \varepsilon)=\{x \mid \rho(p, x)=\varepsilon\}$ is an open set.

Proof of THE Lemma. We need check this only when $S(p, \varepsilon) \neq \varnothing$. In this case necessarily $\varepsilon=1 / m$ for some $m$ in $N$. Let $x \in S(p, \varepsilon), \delta=1 / 2 m(m+1)$ 
and $y \in B(x, \delta)$. Suppose $y \notin S(p, \varepsilon)$. Then, either $\rho(p, y)<\varepsilon$ which implies $\rho(p, y) \leqq 1 /(m+1)$, or $\rho(p, y)>\varepsilon$ and we must have $\rho(p, y) \geqq 1 /(m-1)$ ( $m \geqq 2$ necessarily). In the first case

$$
\begin{aligned}
\rho(p, x) \leqq \rho(p, y)+\rho(y, x) & \leqq \frac{1}{m+1}+\delta \\
& =\frac{1}{m+1}+\frac{1}{2 m(m+1)}<\frac{1}{m}=\varepsilon,
\end{aligned}
$$

a contradiction. If, on the other hand, $\rho(p, y) \geqq 1 / m-1$, then

$$
\frac{1}{m-1} \leqq \rho(p, y) \leqq \rho(p, x)+\rho(x, y)<\frac{1}{m}+\frac{1}{2 m(m+1)} .
$$

But this is true if and only if $1 /(m-1)-1 / m<\frac{1}{2}(1 / m-1 /(m+1))$, again a contradiction. Thus if $y \in B(x, \delta)$ then $y \in S(p, \varepsilon)$. Therefore $S(p, \varepsilon)$ is open. This completes the proof of the lemma.

Suppose now that $x \in \mathrm{Cl}(B(p, \varepsilon)) \backslash B(p, \varepsilon)$. Then $S(p, \varepsilon)$ is a neighbourhood of $x$ not meeting $B(p, \varepsilon)$. Thus $B(p, \varepsilon)$ is closed and open and hence has an empty boundary.

We will now show that, for fixed $\varepsilon>0$, the family $\{B(p, \varepsilon) \mid p \in X\}$ is closure preserving.

Let $A \subseteq X$ be an arbitrary subset. Then

$$
\bigcup_{a \in A} \overline{B(a, \varepsilon)} \subset \overline{\bigcup_{a \in A} B(a, \varepsilon)} \text {. }
$$

Suppose now that $x \in \operatorname{Cl}\left(\bigcup_{a \in A} B(a, \varepsilon)\right) \backslash \bigcup_{a \in A} B(a, \varepsilon)$. Then for all $a \in A$, $\rho(a, x) \geqq \varepsilon$. Thus $\rho(A, x) \geqq \varepsilon$.

Also, there is a sequence $\left\{b_{n}\right\} \subset X$ and a sequence $\left\{a_{n}\right\} \subset A$ such that $\rho\left(x, b_{n}\right)<1 / n$ and $\rho\left(b_{n}, a_{n}\right)<\varepsilon$. Thus $\rho\left(x, a_{n}\right)<\varepsilon+1 / n$ and hence $\rho(A, x)=$ $\varepsilon$.

We will now consider the possible values of $\varepsilon$.

Case 1. Suppose for some $m$ in $N, 1 / m<\varepsilon<1 /(m-1) \leqq 1$. Then $B(x, 1 / m) \cap A=\varnothing$ and $B(x, 1 /(m-1)) \cap A \neq \varnothing$. Thus for all $a \in A$, $\rho(x, a)>1 /(m-1)$ and $\rho(x, A)>\varepsilon$, a contradiction.

Case 2. Suppose now that $\varepsilon=1 / m$ for some $m$ in $N$. Then $\rho(x, A)=$ $1 / m$. Let $\delta=\frac{1}{2}(1 / m+1 /(m+1))$.

If $y \in B(x, \delta) \cap B(a, \varepsilon)$ for some $a$ in $A$ then

$$
\begin{aligned}
\frac{1}{m} & \leqq \rho(x, a) \leqq \rho(x, y)+\rho(y, a) \\
& <\frac{1}{2}\left(\frac{1}{m}-\frac{1}{m+1}\right)+\frac{1}{m+1}<\frac{1}{m}, \text { a contradiction. }
\end{aligned}
$$


Thus $B(x, \delta) \cap\left\{\bigcup_{a \in A} B(a, \varepsilon)\right\}=\varnothing$ contradicting the assumption that $x \in \mathrm{Cl}\left(\bigcup_{a \in A} B(a, \varepsilon)\right)$. Thus

$$
\bigcup_{a \in A} B(a, \varepsilon)=\overline{\bigcup_{a \in A} B(a, \varepsilon)}
$$

and the family is closure preserving.

From these remarks and Nagata's Theorem it follows that Ind $X=0$ proving (i).

"(i) $\Rightarrow$ (ii)". Let $\left\{a_{n}\right\}$ be a strictly monotonically decreasing sequence of real numbers with limit zero. As in Theorem 1 the proof will follow from the proof for $B(m)$. Let $x$ and $y$ be in $B(m)$. Define $f(x, y)$ as in Theorem 1 and set

$$
\begin{aligned}
\rho(x, y) & =a_{f(x, y)} & & \text { when } x \neq y, \\
& =0 & & \text { otherwise. }
\end{aligned}
$$

Let $x, y$ and $z$ be distinct points in $B(m)$. Then either $f(x, y) \geqq f(x, z)$ or $f(x, y)<f(x, z)$ in which case $f(x, y)=f(y, z)$. Thus in either case $f(x, y) \geqq$ $\min \{f(x, z), f(y, z)\}$. Therefore $\rho(x, y) \leqq \max \{\rho(x, z), \rho(y, z)\}$ and thus $\rho$ satisfies the triangle law. The other metric axioms and the fact that $\rho$ is compatible with the product topology on $B(m)$ are easily checked. This completes the proof of the theorem.

CONCLUDING ReMARKS. If $F \subseteq[0, \infty)$ has 0 as its only cluster point $F$ is a countable set and we may label the points of $F \cap[0,1], \alpha_{1}>\alpha_{2}>\alpha_{3}>\cdots>$ 0 . The sequence $\left\{a_{n}\right\}$ is monotonically decreasing and has limit 0 . Thus we have shown:

Corollary. The space $(X, \tau)$ has $(\check{C} e c h)$ dimension zero if and only if there exists a metric for $X$, generating the topology $\tau$, having values in $R^{+}$ with 0 as the only cluster point of those values.

\section{REFERENCES}

1. J. de Groot, On a metric that characterizes dimension, Canad. J. Math. 9 (1957), 511-514. MR 19, 874.

2. J. Nagata, On a relation between dimension and metrization, Proc. Japan Acad. 32 (1956), 237-240. MR 19, 156.

3. - Two theorems for the n-dimensionality of metric spaces, Compositio Math. 15 (1963), 227-237. MR 29 \#1617.

4. L. Janos, A metric characterization of zero-dimensional spaces, Proc. Amer. Math. Soc. 31 (1972), 268-270.

5. K. Broughan and M. Schroder, Variations on a metric theme, Report \#14, Univ. of Waikato Research, 1972.

6. K. Morita, Normal families and dimension theory for metric spaces, Math. Ann. 128 (1954), 350-362. MR 16, 501.

Department of Mathematics, University of Waikato, Hamilton, New Zealand 